\title{
A Greedy Routing Algorithm for Deep Sea without Location Information
}

\author{
Hongning $\mathrm{Hu}^{\mathrm{a}, \mathrm{b}}$, Zhong Liu \\ Naval University of Engineering, Wuhan 430033, China \\ asynorsmith@hotmail.com, bhuhongning@hotmail.com
}

\begin{abstract}
Inspired by the biological process of "shark foraging", this paper proposes a novel greedy routing algorithm based on the "odor diffusion mechanism" and "shark greedy foraging mechanism". The algorithm doesn't need the location information, and it can transmit packets to the target node quickly, which has strong adaptability to the deep sea sparse network.
\end{abstract}

Keywords: Deep sea sparse network; Odor diffusion mechanism; Shark greedy foraging mechanism; Location information.

\section{Introduction}

The deep sea network has the characteristics[1,2] of sparse node distribution, uncertain topology structure and large underwater acoustic communication delay, so many traditional routing algorithms are not suitable for it. The deep sea network model is shown in Fig.1. Although the DREAM [3] algorithm can solve the "hole" problem [2,4] faced by sparse network, as shown in Fig.2, it will produce a large number of message copies and increase the load of the whole network. The present technology can't meet the requirements of getting the node's location from deep sea [5, 6], so DREAM does not have the practical operability.

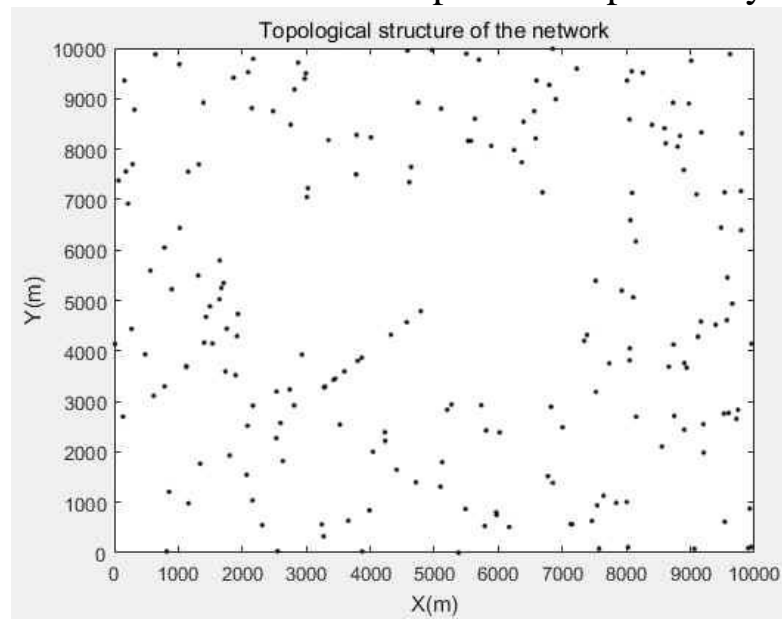

(a) Distribution of nodes in the network

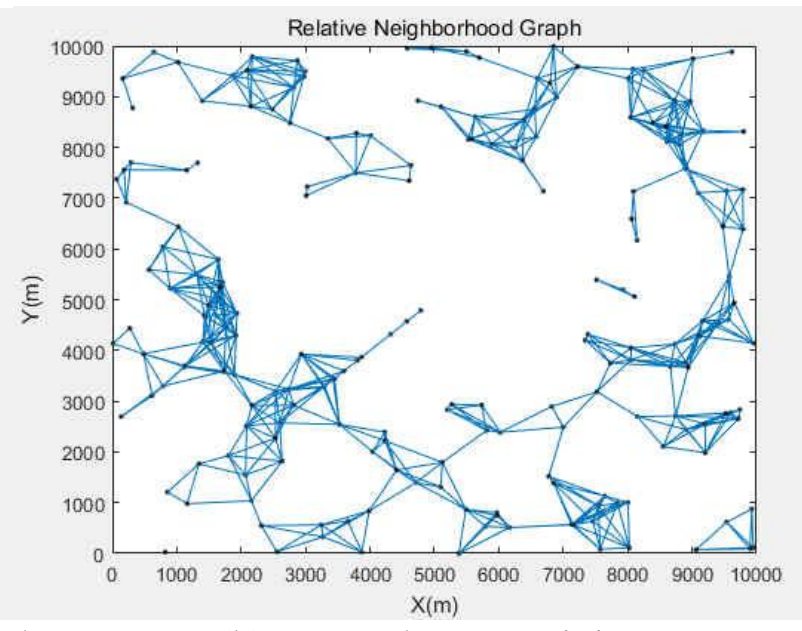

(b) Network connectivity

Fig. 1 The Network Model

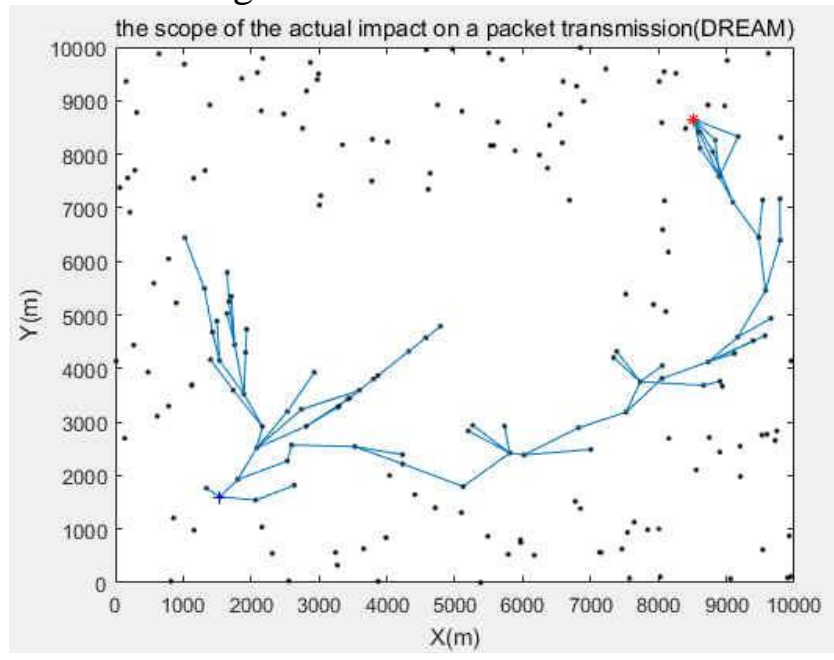

Fig. 2 The actual propagation of packets in DREAM 
In this paper, we propose a greedy routing algorithm that does not need the location information of nodes, which is inspired by the biological process of "Shark foraging". In the algorithm, each super-node is assumed to be a "injured fish", interval time to release their own "odor". The packages are the "shark", along the "odor" quickly catch the target super-node, and then complete the transmission of information. As long as there is a communication path between the two super-node, the algorithm can ensure that the data packets are sent to the target node with the least number of hops required.

\section{Description of The Novel Greedy Algorithm}

\subsection{Network Model.}

There are two kinds of nodes in the network, which are super-node and normal-node. Among them, only super-node can produce information, normal-node is responsible for the forwarding of information. All nodes have a neighbor table who is updated and maintained by using S-Frames received. The structure of the neighbor table is shown in Fig.3.

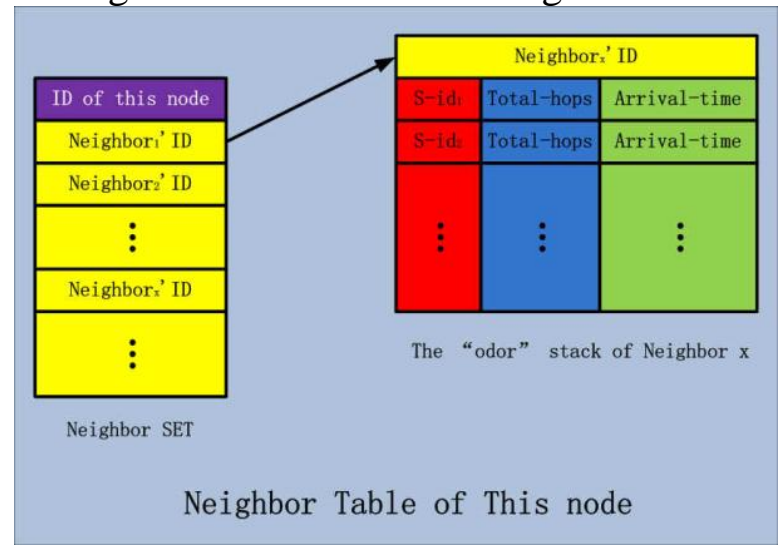

Fig. 3 Structure of the Neighbor Table

Figure 3 shows that the neighbor table consists of the neighbor set and the "odor" stack. The neighbor set is used to store all known neighbor node's id in one hop range, and the "odor" stack save all "super-node" odor concentration, which is determined by "Total-hops" and "Arrival-time".

\subsection{Odor Diffusion Mechanism.}

The odor diffusion of super-nodes are realized through the forwarding of S-Frame in the network.

(1)S-Frame.

The S-Frame is generated by a super-node, it mainly has two functions, update the node's neighbor node table and leave the "odor concentration" of super-node. The data structure of S-Frame is shown in Fig.4.

\section{S-id $\quad$ Last-hop-id SET $\quad$ Total-hops Arrival-time}

Fig. 4 The data structure of S-Frame

The S-id is a super-node's id who has generated the S-Frame. The Last-hop-id SET is an array used to store id of all nodes arrived, the structure and the update process as shown in Fig.5. The Total-hops represents the number of times it has been forwarded so far, and its value is the same as the total number of ids in Last-hop-id SET. The Arrival-time saves the time of S-Frame received by last hop node.

(2)Odor Diffusion Mechanism.

When a node receives an S-Frame, it first determines whether the S-Frame is new or not, and the basis of the judgment is to check whether the node's id is included in Last-hop-id SET of the S-Frame. If the Last-hop-id SET does not contain the node's id, the node will use the S-Frame to update its Neighbor Table, and then add its own id into Last-hop-id SET, update and forward the S-Frame. If it is the opposite, the node will drop the S-Frame directly. The specific process of odor diffusion mechanism is shown in Fig.6. 


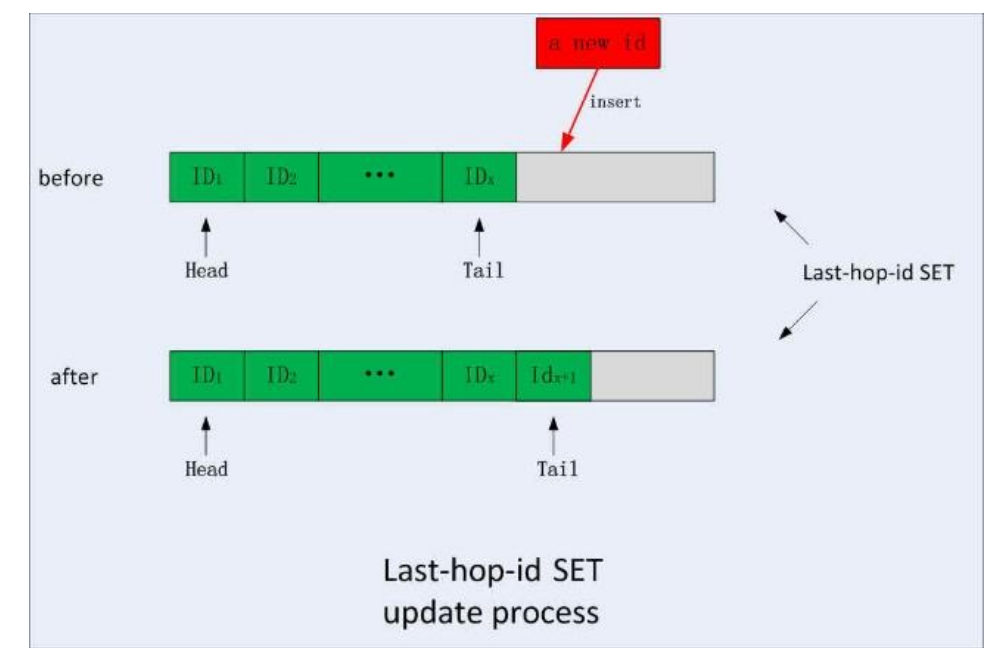

Fig. 5 The last-hop-id SET update process

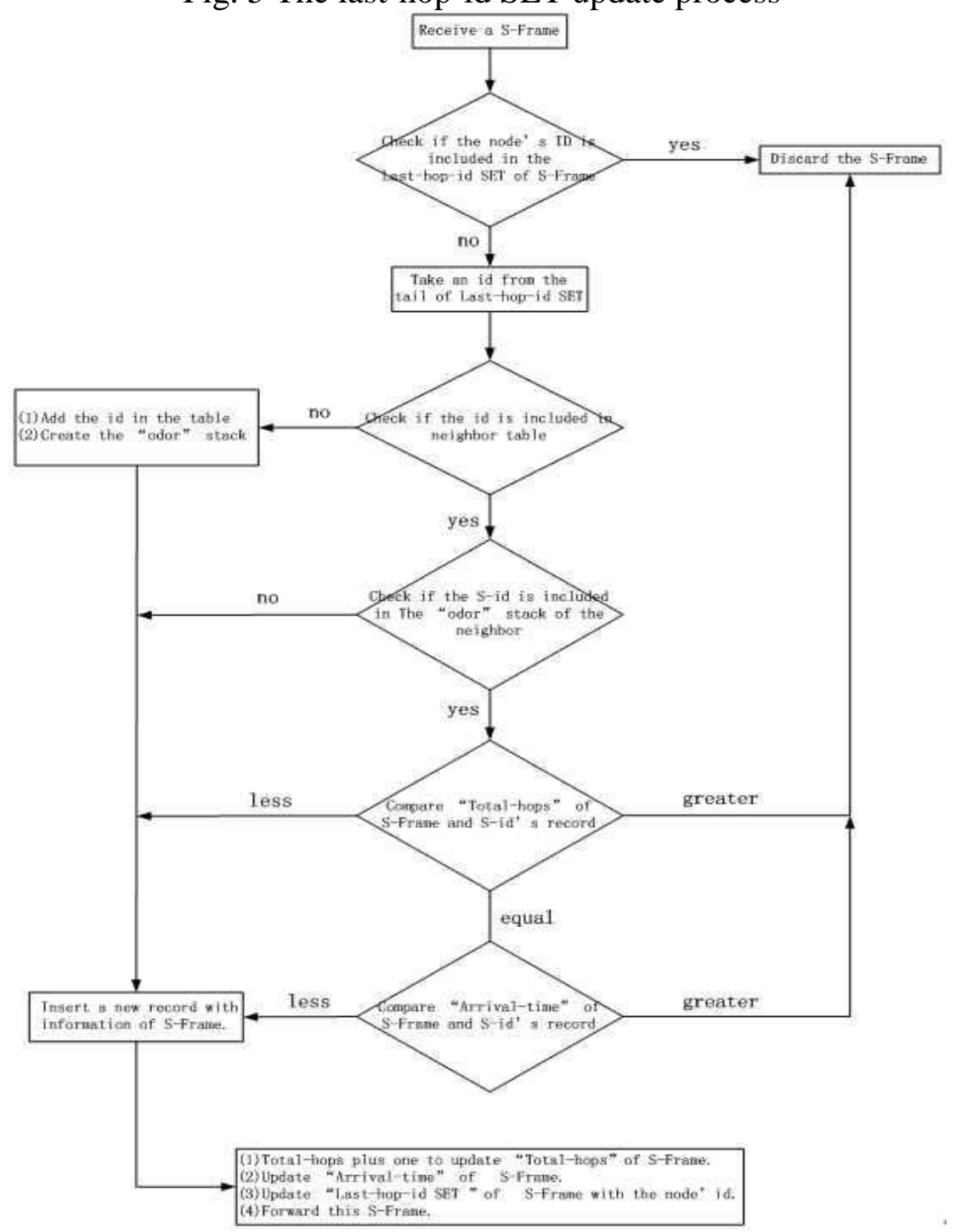

Fig. 6 The Specific Process of Odor Diffusion Mechanism

\subsection{Shark Foraging Mechanism.}

(1)The "shark" packet.

The communication data is transmitted through the "shark" packet. A shark packet is divided into a head and a trunk, and the head is used to store all ids of the target super-node and the next hop node, and the trunk is used for storing the communication data. The structure of the shark packet is shown in Fig.7. 


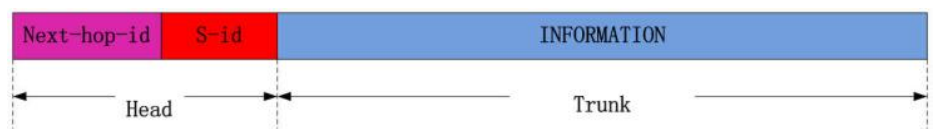

Fig. 7 The Structure of "shark" packet

(2)Determination rules of odor concentration.

The hop is held essential with time-consuming as a supplement in the determination rules of odor concentration. As shown in Fig.8, in case 1, the node E's hops was less than the node C's, so the odor concentration of E was higher than C's; in case 2, the E's time time-consuming is less than C's, so C's odor concentration was higher than D's; in case 3, who owns a higher odor concentration between C and $\mathrm{D}$ is based on a random selection.

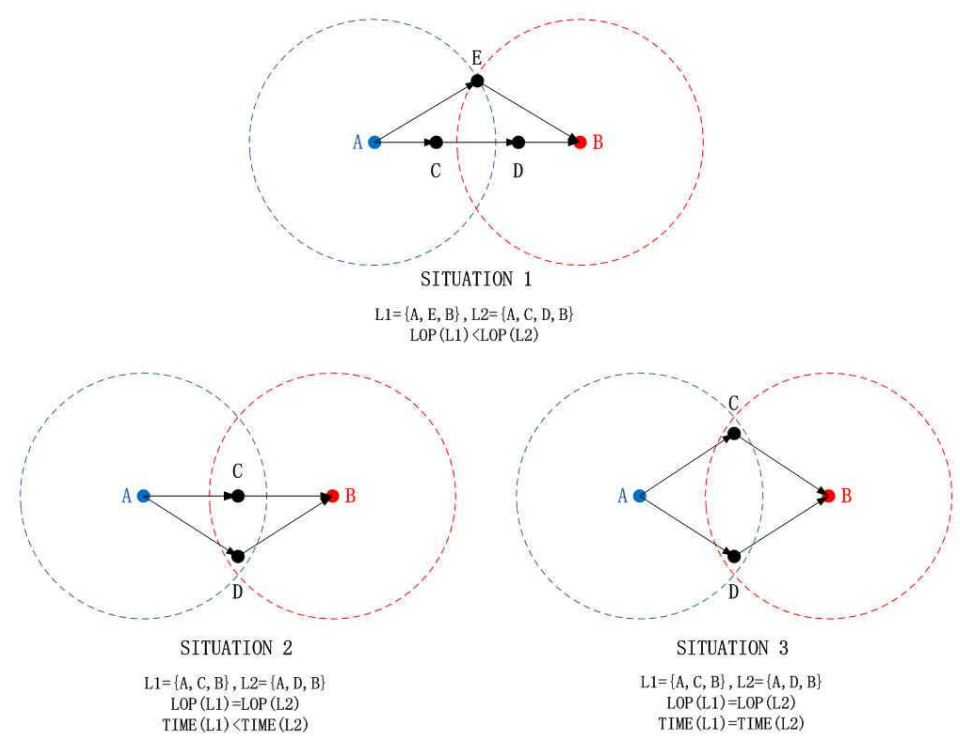

Fig. 8 Determination rules of odor concentration

(3)Greedy foraging process.

The greedy foraging process is embodied in the process of receiving and processing the "shark" packets through the network nodes, the basic process is as follows:

Step 1: After receiving the "shark" packet, the node first check whether it's own id is in agreement with the S-id in the head of the packet. If the two id are in agreement, and then receives the packet and end the communication. Otherwise, turn to Step 2;

Step 2: Continue to check whether the Next-hop-id is the same as the node's id, if not the same, then directly discards the packet. Otherwise turn to Step 3;

Step 3: Search the id who has the highest odor concentration in it's neighbor table, and use the id to update the Next-hop-id of the "shark" packet.

\section{Simulation Experiment}

The network model and simulation parameters of the literature [2] are used for reference. The deep sea network is very sparse, in order to be closer to the real scene, the number of normal-node in the model is reduced to 200, and its topology structure is shown in Fig. 1.

The experimental scenario is: a super-node "blue +" randomly sends 100 packets to another super-node "red +" within an hour.

The first, twentieth, fiftieth and 100th times of the new algorithm's data packet transmission are presented in Fig. 9. From the graph, we can see that the packet has been moving forward to the densest node, and the propagation path of the packet is the shortest path. Although super-node has been in motion, as long as its neighbor node who has the highest odor concentration among other neighbors are still in the normal communication range, which can be achieved by setting the interval time of odor diffusion mechanism, the new algorithm can ensure the smooth completion of communication. 
The propagation of DREAM is shown in Fig. 9. DREAM has good robustness, which can guarantee the data packets to be delivered to the target super-node successfully, but the routing overhead is also high. With extension of communication time, the network is filled with a large number of packet copies, which leads the collision probability of packets and the propagation delay goes rising sharply. After 4 times of communication, the network load achieves a stable value, then the communication delay is also stable at around 49s, as shown in Fig. 10.

Figure 10 shows the performance comparison of two algorithms for network load and propagation delay. In the new algorithm, the "shark" data packets are transmitted only along the shortest path, which does not generate duplicate packet copies, so the network load and communication delay have been stable at a small value.

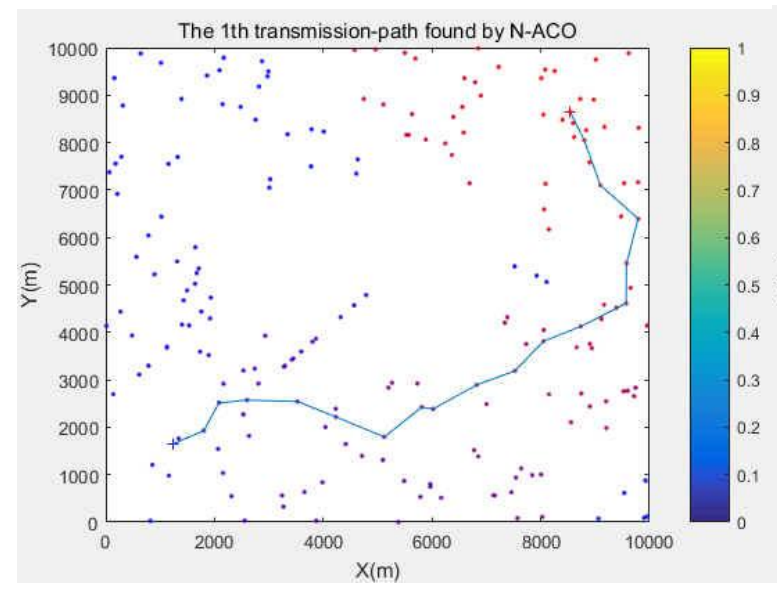

(a)The 1th transmission

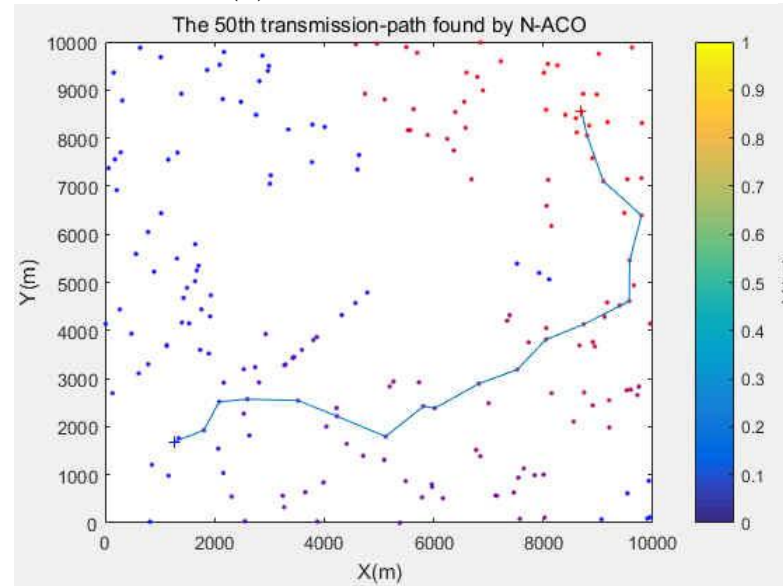

(c) The 50th transmission

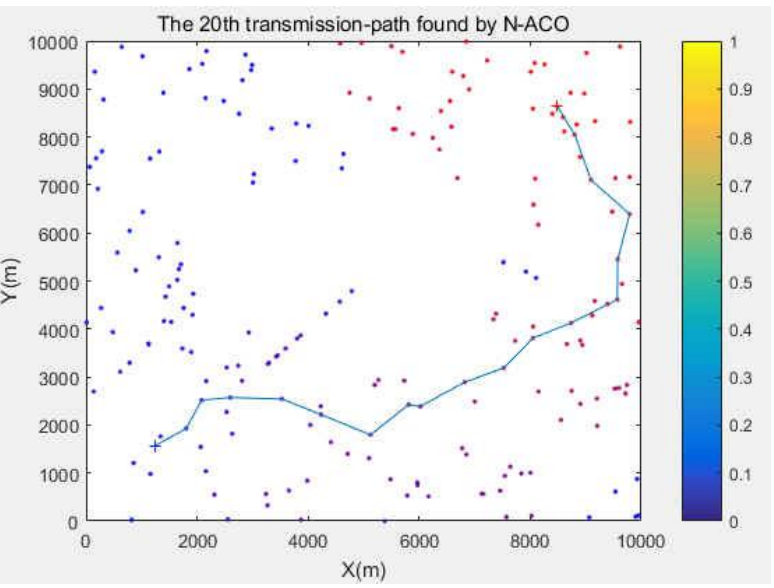

(b) The 20th transmission

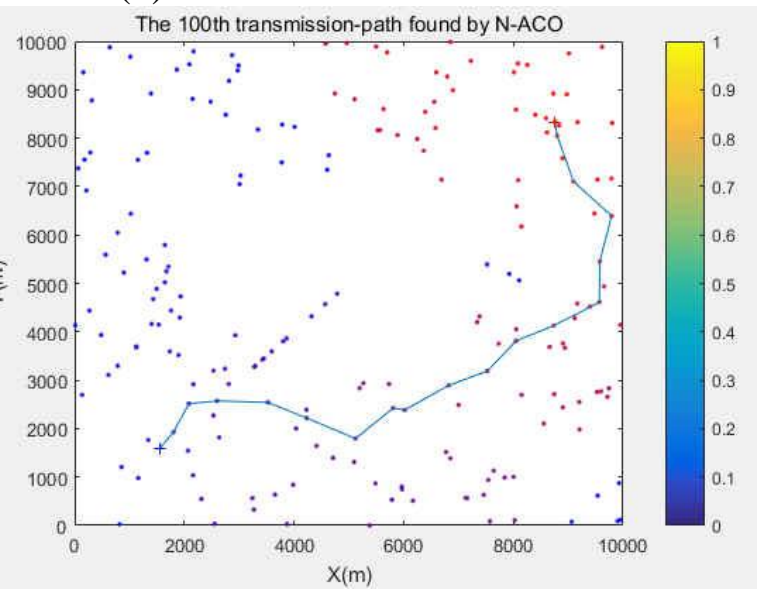

(d) The 100th transmission

Fig.9 The actual propagation of the new algorithm

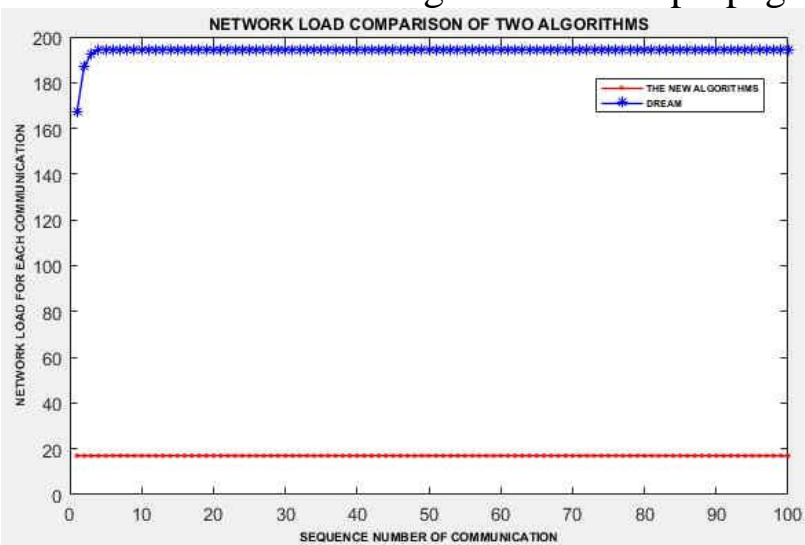

(a) Network Load

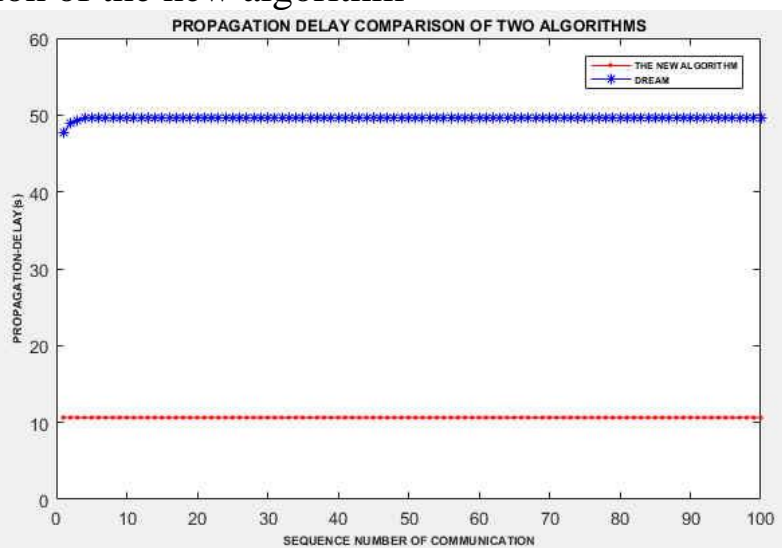

(b) Propagation Delay

Fig. 10 Performance Comparison between the new algorithm and DREAM 


\section{Conclusion}

In this paper, a novel greedy routing algorithm is proposed, which can transmit packets to the target node quickly without node's Location information by only relying on the "odor diffusion mechanism" and "shark greedy foraging mechanism". Simulation results show that the new algorithm can well solve the sparse hole problem in deep sea network, and achieves excellent performance in decreasing propagation delay and reducing network load. Therefore, the algorithm has strong adaptability to the deep sea network, and it can be operated in reality.

\section{Acknowledgements}

This paper was supported by the Natural Science Foundation of Naval Engineering University (No.HGDKYJGZX15007).

\section{References}

[1]. Ian F Akyildiz, Dario Pompili, Tommaso Melodia. Underwater Acoustic Sensor Networks: Research Challenges. Ad Hoc Networks. Vol. 3 (2005) No. 3, p. 257-279.

[2]. Hu HongNing, Liu Zhong, Yang Bin. BFDREAM: A new routing protocol for deep sea acoustic network. 2010 IEEE 10th INTERNATIONAL CONFERENCE ON SIGNAL PROCESSING PROCEEDINGS (ICSP2010). Beijing: Springer Verlag, 2010, p. 2377-2381.

[3]. Stefano B, Imrich C, Violet R. S, et al. A distance routing effect algorithm for mobility (DREAM). Proceedings of the 4th annual ACM/IEEE international conference on Mobile computing and networking. DALLAS TEXAS, 1998, p.76-84.

[4]. ZHANG Hengyang, LI Yingying, LIU Yunhui. Research of position-based routing for wireless sensor networks. Application Research of Computers. Vol. 25 (2008) No. 1, p. 18-21, 28.

[5]. J Partan, J Kurose, B. N. Levine. A survey of practical issues in underwater networks. In Proceeding of the First workshop on Underwater networks (WuWNet). Los Angeles, CA, USA, 2006, p. 17-24.

[6]. WANG Jing, CHEN Jan-feng, ZHANG Li-jie, et al. Underwater sensor networks. Technical Acoustics. Vol.28 (2009) No.1, p. 89-95. 